\title{
Association of Heart Failure with Preserved Ejection Fraction and Components of Metabolic Syndrome
} \author{
Dogaru $\mathrm{G}^{3}$ \\ $13^{\text {rd }}$ Internal Medicine Clinic, Faculty of Medicine, University of Medicine and Pharmacy, Tirgu-Mures, Romania \\ $21^{\text {st }}$ Gastroenterology Clinic, Faculty of Medicine, University of Medicine and Pharmacy, Tirgu Mures, Romania \\ 3 Department of Nephrology, University of Medicine and Pharmacy, Tirgu-Mures, Romania \\ 4 Department of Urology, University of Medicine and Pharmacy, Tirgu-Mures, Romania \\ 5 Department of Physiology, University of Medicine and Pharmacy, Tirgu-Mures, Romania
}

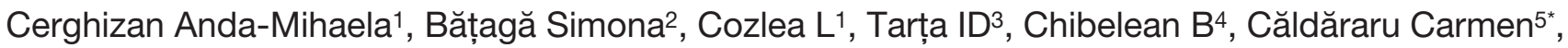

\begin{abstract}
The aim of the current study was to find any possible associations between elements of metabolic syndrome and echocardiographic characteristics (grade of diastolic dysfunction) in normoponderal and overweight patients with heart failure with preserved ejection fraction.

A retrospective observational analytical study was performed on 130 patients presenting heart failure with documented ejection fraction over $50 \%$. They were divided into two groups based on their body mass index. The first group included 56 normal weight patients and the second group included 74 overweight patients. Elements of the metabolic syndrome analysed in the current study were arterial hypertension, high triglyceride levels, low HDL-cholesterol and diabetes.

None of the components of metabolic syndrome alone had a role in the evolution of diastolic dysfunction in either group. Three or four elements present in obese patients were negatively associated with grade 2 diastolic dysfunction, high values of blood pressure (over 180/110 $\mathrm{mmHg}$ ) were more often encountered in obese patients with first and second grade of diastolic dysfunction.
\end{abstract}

Keywords: diastolic dysfunction, metabolic syndrome, body mass index.

Received: 23 June 2015 / Accepted 03 August 2015

\section{Introduction}

A generally accepted definition of heart failure with preserved ejection fraction (HF-PEF) developed by Zale and Brutsaert, states that this is "a clinical syndrome characterized by the symptoms and signs of heart failure, a preserved ejection fraction (EF), and abnormal diastolic function" [1].

The prevalence of this syndrome in general population, according to some studies ranges between $30 \%$ to $50 \%$ of patients with heart failure $[2,3]$. The annual mortality rate of patients presenting HF-PEF is $5-8 \%$ lower than in patients with systolic dysfunction, and it depends on the associated comorbidities [1].

Patients with heart failure (HF) frequently pesent a high number of comorbidities. In one study conducted by Senni M and Redfield MM on 216 patients with HF, fifty-nine of them had preserved systolic function and also presented valve disease, atrial fibrillation, coronary heart disease, hypertension or metabolic syndrome (MetS) $[4,5]$.

According to the National Cholesterol Education Program's Adult Treatment Panel III metabolic syndrome is composed of the following 6 elements: abdominal obesity, atherogenic dyslipidaemia, elevated blood pressure, insulin resistance or impaired glucose tolerance, proinflammatory state and prothrombotic state [6].
In need for a simpler and easier definition to apply by clinicians worldwide, in 2006 the International Diabetes Federation defined metabolic syndrome as central obesity accompanied by any of the two following elements: elevated serum triglycerides $(\geq 1.7 \mathrm{mmol} / \mathrm{l})$, low HDL cholesterol $(<40 \mathrm{mg} / \mathrm{dl}$ in male, $<50 \mathrm{mg} / \mathrm{dl}$ in female), elevated blood pressure $(>130 / 85 \mathrm{mmHg})$, fasting hyperglycaemia $(>100$ $\mathrm{mg} / \mathrm{dl}$ ) or diagnosed type 2 diabetes [7]. Components of the MetS such as high blood pressure, diabetes and low HDL cholesterol levels have been identified as major and independent cardiovascular risk factors [8].

The E/A ratio is efficient in assessing diastolic dysfunction (DD). The normal value is between 1 and 2 which means that $\mathrm{E}$ wave is taller than $\mathrm{A}$ wave and the ventricle fills passively. The E/A ratio lower than 1 characterises the first grade of diastolic dysfunction (DD1 - altered relaxation pattern) and it consists in less blood in the ventricle during early filling and more blood is present in the atria during contraction, thus a larger $\mathrm{A}$ wave than $\mathrm{E}$ wave. In grade 2 of diastolic dysfunction (DD2 - pseudonormalized pattern) the E/A ratio is between 0.8 and 1.5 and it's characterized by high left atrial pressure. The E/A ratio higher than 2 implies a growth of the gradient between the left atria and ventricle during diastole, thus $\mathrm{E}$ wave becomes taller and the A wave smaller, this defining the third grade of diastolic dysfunction (DD3 - restrictive pattern) [9].

The purpose of this study was to find possible associations between elements of metabolic syndrome and echocardiographic characteristics (grade of diastolic dysfunc- 
tion) in a group of normal weight patients compared to a group of overweight patients with HF-PEF.

\section{Material and method}

A retrospective observational analytical study was performed on 130 patients hospitalized the $3^{\text {rd }}$ Internal Medicine Clinic of Tirgu Mures between $1^{\text {st }}$ of July $2013-1^{\text {st }}$ of January 2014. The main inclusion criteria was a documented ejection fraction higher than $50 \%$, in the presence of clinical signs of heart failure.

The left ventricular ejection fraction (LVEF), the grade of peak mitral Doppler E/A ratio and the left ventricular mass $\left(\mathrm{g} / \mathrm{m}^{2}\right)$ from the echocardiographic evaluation were collected for all the study population.

Patients were divided in 2 groups (Table I) according to the World Health Organization's classification of body mass index (BMI).

Other followed elements of the MetS were:

- documented high blood pressure (HBP) by grades [10] as follows

- grade 1 - systolic BP 140-159 mmHg, diastolic BP 90-99 mmHg;

- grade 2 -systolic BP 160-179 mmHg, diastolic BP 100-109 mmHg;

- grade 3 - systolic $\mathrm{BP} \geq 180 \mathrm{mmHg}$, diastolic $\mathrm{BP} \geq$ $110 \mathrm{mmHg}$.

- elevated triglyceride levels $-\geq 1.7 \mathrm{mmol} / \mathrm{l}$;

- low HDL-cholesterol - <40 mg/dl at male, $<50 \mathrm{mg} /$ $\mathrm{dl}$ at female;

- type 2 diabetes mellitus.

Table I. Body mass index classification

\begin{tabular}{lccc}
\hline Group & Classification & BMI $(\mathrm{kg} / \mathrm{m} 2)$ & Patients \\
\hline 1 & Normal & $18.50-24.99$ & 56 \\
\hline \multirow{3}{*}{2} & Pre-obese & $25.00-29.99$ & 17 \\
& Obese class I & $30.00-34.99$ & 40 \\
& Obese class II & $35.00-39.99$ & 13 \\
& Obese class III & $\geq 40.00$ & 4 \\
\hline
\end{tabular}

We assembled a patient database using Excel 2010 software and Epi Info software was used for statistical analysis. Fisher's exact test, Chi square Yates corrected and Chi square tests were used, with a confidence level of $95 \%$; $p<$ 0.05 was considered statistically significant.

Continuous variables are reported as mean \pm standard deviation (SD) and categorical variables as observed number of patients.

\section{Results}

From the total group of selected patients, 74 had a BMI higher than 25 . In the selected cohort, we had 61 male $(46.92 \%)$ and 69 women $(53.08 \%)$ with a mean age of 62 \pm 9 years.

The patients were divided into 2 groups, based on their BMI, with group characteristics shown in Table II.
Table II. General characteristics of groups.

\begin{tabular}{lcc}
\hline Group Characteristics & Group 1 & Group 2 \\
\hline $\mathrm{n}$ & 56 & 74 \\
Sex (male) & $32(57.14 \%)$ & $29(39.19 \%)$ \\
Mean age (years) & $63 \pm 9$ & $62 \pm 9$ \\
HBP & $53(94.64 \%)$ & $71(95.95 \%)$ \\
High triglyceride levels & $14(25.00 \%)$ & $29(39.19 \%)$ \\
Low HDL-cholesterol & $25(44.64 \%)$ & $59(79.73 \%)$ \\
Diabetes mellitus & $10(17.86 \%)$ & $26(35.14 \%)$ \\
Mean LVEF (\%) & $58.23 \pm 3.67$ & $58.68 \pm 4.02$ \\
Mean E/A ratio & $0.91 \pm 0.42$ & $0.86 \pm 0.47$ \\
LV mass (g) & $184.36 \pm 56.45$ & $192.8 \pm 63.66$ \\
HBP - high blood pressure; LVEF- left ventricular & \\
ejection fraction; LV- left ventricle; & & \\
& &
\end{tabular}

Analysing the two groups regarding the presence of hypertriglyceridemia, low HDL-cholesterol levels, diabetes and the association with different grades of diastolic dysfunction, there was no statistically significant association. High triglyceride levels don't have a proven role in development and evolution of different grades of diastolic dysfunction in either group ( $\mathrm{p}$ value 0.14 and $0.76-$ no statistical significance). Low levels of HDL-cholesterol do not associate statistically with diastolic dysfunction in normal or obese patients $(p=0.75, p=0.72)$. The presence of diabetes mellitus doesn't affect the grade of diastolic function, ( $p$ value 0.21 in normal BMI patients, and $p=0.17$ in patients with $B M I \geq 25 \mathrm{~kg} / \mathrm{m}^{2}$ ).

The prevalence of diastolic dysfunction in the total cohort was $78.46 \%$ grade $1,18.46 \%$ grade 2 and $3.08 \%$ grade 3 . The comparison between the distributions of diastolic dysfunction grades in the two groups is shown in Figure 1.

In the first group - normal weight patients - there were no statistically significant associations. Fourty three patients (representing almost $80 \%$ ) had first grade of diastolic dysfunction (E/A ratio < 1 ) out of which $24(55,81 \%)$ presented grade 2 hypertension.

In the second group - overweight patients - there was a positive statistically significant association between first grade diastolic dysfunction and grade 2 hypertension $(p=0.042$, OR 0.24; [0.06-094]). A negative statistical as-

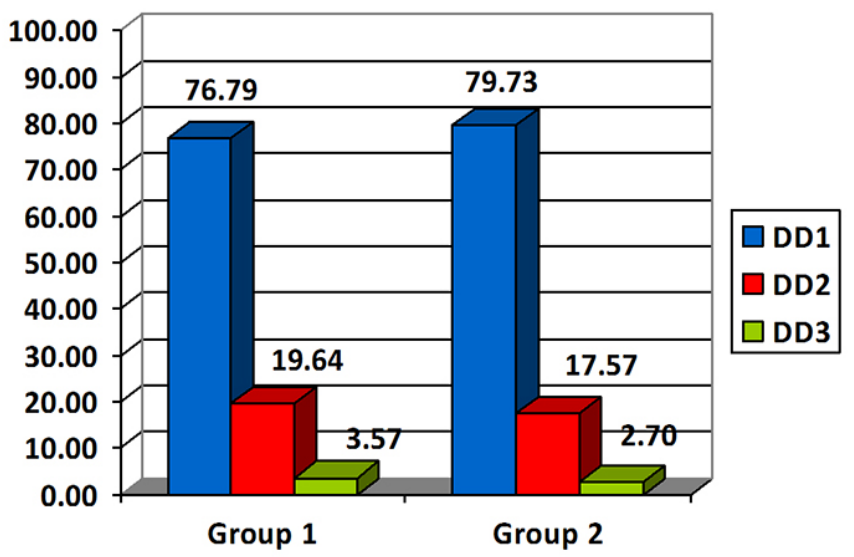

Fig. 1. Distribution of diastolic dysfunction (E/A ratio) in the two groups 
sociation between grade 3 hypertension and the first and second grade of diastolic dysfunction was found. ( $\mathrm{p}=0.006$, OR 11.03; [1.36-89.34] and $\mathrm{p}=0.024$, OR 3.11 [1.437.39] respectively).

Blood pressure grading among our two studied patient groups is shown in Figure 2.

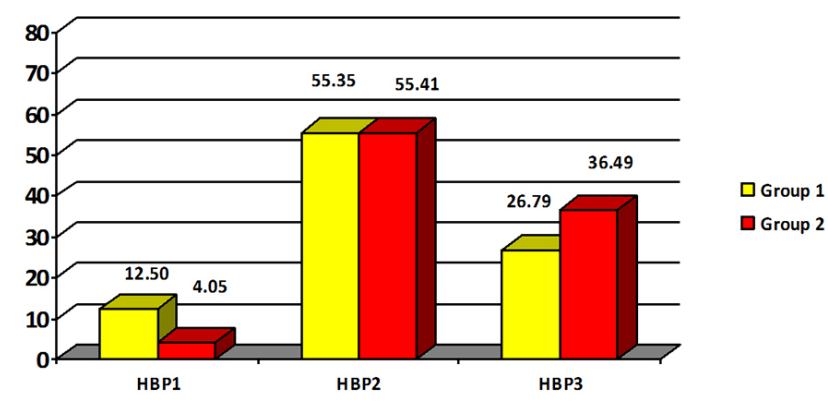

HBP1- High blood pressure stage 1

HBP2- High blood pressure stage 2

HBP3- High blood pressure stage 3

Fig. 2. Blood pressure grades in normoponderal and overweight patients

Comparing the number of elements of MetS with the grade of DD in normal weight patients, $8(14.29 \%)$ of them had 2 elements, 25 (44.64\%) of the patients had 3 elements, and $2(3.57 \%)$ patients had all 4 elements (arterial hypertension, high triglycerides level, low HDL cholesterol and diabetes), with no statistically significant association between the number of elements and the grade of diastolic dysfunction.

In the second group (overweight patients) 27 (36.49\%) had 2 elements of MetS, $23(31.08 \%)$ had 3 elements and $13(17.57 \%)$ had 4 elements. A statistically significant association was found between patients with 3 and 4 elements of MetS and diastolic dysfunction grade $2(\mathrm{p}=0.011)$.

\section{Discussion}

We tried to establish the role of elements of MetS in normoponderal and obese patients with diastolic dysfunction.

Low HDL-cholesterol levels, high triglycerides, arterial hypertension and diabetes mellitus are amongst the most feared risk factors for heart disease, along with advanced age and smoking [11]. None of the components of metabolic syndrome alone had a role in the evolution of diastolic dysfunction, regardless of the weight.

In a recent study an increase in the left atrial diameter was associated with MetS in DD patients [12]. Our results revealed that a combination of three or four components of the MetS in overweight patients had been statistically associated with a worsening in the DD to second grade, characterised by high left atrial pressure.

In a similar study, but conducted only on obese patients, the authors [4] found similar results with our study, regarding the number of characteristics of MetS, thus patients with 3 characteristics (including obesity) represented $40.3 \%$ vs. $36.5 \%$ in the current study, patients with 4 characteristics represented $38.9 \%$ vs. $31.1 \%$ and patients with all the characteristics of the MetS were at $20.8 \%$ vs. $17.6 \%$ in our study. We went further and tried to find a correlation in obese hypertensive patients, between the grade of arterial hypertension and the grade of diastolic dysfunction, and we found that high blood pressures (values over 180/110 $\mathrm{mmHg}$ ) represent a risk factor for development of first grade diastolic dysfunction and progression to second grade of diastolic dysfunction.

Arterial hypertension, along with diabetes, hypertriglyceridemia and increased waist circumference (central obesity) are associated with the grade of diastolic dysfunction, according to some recently published studies $[4,13]$. However, Aksoy S et al concluded in their 2014 published study conducted on a small number of patients (60 patients) that diastolic dysfunction may develop in patients with MetS in the absence of hypertension [14].

\section{Conclusion}

The presence of 3 or 4 elements of metabolic syndrome in overweight patients is associated with higher grades of diastolic dysfunction, but each element taken separately did not prove to play a role in the progression of diastolic dysfunction.

High blood pressure values (over 180/110 $\mathrm{mmHg}$ ) represent a risk factor in obese patients for the development of first or second grade of diastolic dysfunction.

Low HDL-cholesterol and hypertriglyceridemia were not associated with diastolic dysfunction in our study.

\section{References}

1. Zile MR, Brutsaert DL. New concepts in diastolic dysfunction and diastolic heart failure: part I: diagnosis, prognosis, and measurements of diastolic function. Circulation. 2002;105:1387e93.

2. Bonow RO, Udelson JE. Left ventricular diastolic dysfunction as a cause of congestive heart failure. Mechanisms and management. Ann Intern Med. 1992;117:502-510.

3. Vasan RS, Benjamin EJ, Levy D. Prevalence, clinical features and prognosis of diastolic heart failure: an epidemiologic perspective. J Am Coll Cardiol. 1995;26:1565-1574.

4. Penjašković $D$, Sakač $D$, Dejanović $J$ et al. Left ventricular diastolic dysfunction in patients with metabolic syndrome. Med Pregl. 2012;65(12):18-22.

5. Senni M, Redfield MM. Heart failure with preserved systolic function. A different natural history? J Am Coll Cardiol. 2001;38:1277-1282.

6. Third report of the National Cholesterol Education Program (NCEP) expert panel on detection, evaluation, and treatment of high blood cholesterol in adults (Adult Treatment Panel III). Final report. Circulation. 2002;106:3143-3421.

7. Alberti KG, Zimmet P, Shaw J. Metabolic syndrome: a new world-wide definition. Diabet Med. 2006;23(5):469-480.

8. Grundy SM, Pasternak R, Greenland $\mathrm{P}$ et al. Assessment of Cardiovascular Risk by Use of Multiple-Risk-Factor Assessment Equations. Circulation. 1999;100:1481-1492.

9. William C. Little, Jae K. Oh. Controversies in Cardiovascular Medicine. Echocardiographic Evaluation of Diastolic Function Can Be Used to Guide Clinical Care. Circulation. 2009;120:802-809.

10. Mancia G, Fagard R, Narkiewicz K et al. 2013 ESH/ESC Guidelines for the management of arterial hypertension. The Task Force for the management of arterial hypertension of the European Society of Hypertension (ESH) and of the European Society of Cardiology (ESC). European Heart Journal. 2013;34:2159-2219.

11. Jousilahti P, Vartiainen E, Tuomilehto J et al. Sex, Age, Cardiovascular Risk Factors, and Coronary Heart Disease. A Prospective Follow-Up Study of 14786 Middle-Aged Men and Women in Finland. Circulation. 
1999;99:1165-1172.

12. Ayalon N, Gopal DM, Mooney DM et al. Preclinical left ventricular diastolic dysfunction in metabolic syndrome. Am J Cardiol. 2014;114(6):838842

13. Rashid Khan A, Qaisar Khan M. Association and pattern of diastolic dysfunction in patients of metabolic syndrome. J Ayub Med Coll Abbottabad. 2008;20(2):70-75.

14. Aksoy S, Durmus G, Ozcan S et al. Is left ventricular diastolic dysfunction independent from presence of hypertension in metabolic syndrome? An echocardiographic study. J of cardiol. 2014;64(3):194-198. 